\title{
Predicators for Marital Cohesiveness in Three-generation Family
}

\author{
Taku Kobayashi $^{1)}$, Norimasa Itakura ${ }^{2)}$, Takaaki Usami ${ }^{2)}$, Mio Sato ${ }^{2)}$, \\ Airi Shimizu $^{2)}$, and Koubun Wakashima ${ }^{2)}$ \\ 1) School counselor of Sendai-City \\ ${ }^{2)}$ Graduate School of Education, Tohoku University
}

\begin{abstract}
The purpose of this study was to unfold the complicated mutual link in the family. Specifically, we report how marital relationship relates with other dyads in the three-generation family (first generation (G1) was referred as grandparents, second (G2) as parents, and third (G3) as child's generation) with Inventory for Character of Intra-Inter Generation in Kinship -"ICHIGEKI"-. Result of multi regression analyses on wife-husband cohesiveness in multigenerational family revealed that seven dyadic relationships are predictive of marital cohesiveness in three-generation family.
\end{abstract}

$\underline{\text { KEY WORDS: Multi-generation Family, Marital Relationship, Cohesiveness, ICHIGEKI }}$

Family is a system based on complex intercommunication. Therefore, it is not easy to understand how a specific dyad relates to others. Wakashima, et al.(2010) showed the empirical way to examine the association between dyads in the family, but two limits can be pointed out to their methodology. First, it is difficult to reach any conclusions because they obtained excessive amounts of result. Second, there are large amount of potentially predictive factors to examine the dyadic relationship in multigenerational family. Hence, the analysis should be performed for participants of each family type.

To overcome the limitations, we focus on the

CORRESPONDENCE TO: KoBAYASHI, Graduate School of Education, Tohoku University, 27-1 Kawauchi, Aoba-ku, Sendai-city, 980-8576, Japan.

e-mail: t_5884@live.jp most core factor, marital relationship for an assessment of three-generation family (first generation; G1 was referred as grandparents, second generation;G2 as parents, and third geberation;G3 as child's generation). It is important to recover marital relationship for clinical treatments, because this relationship relate to not only themselves mental health but also the other family members' mental health, especially child's mental health (Kawashima, et al. 2008). Therefore, to explore predicators for marital relationship is useful for clinician who helps families.

\section{Methods}

\section{Participants}

Research participants were Mother from G2. If there were two or more children (G3) in a family, the participants rated the family relationship with first-born child.

Measures 
We assessed dyadic cohesiveness as family member's inter-relationship

in multi-generational family with cohesiveness. Cohesiveness-Scale was applied from one of the subscales of "Inventory for Character of Intra-Inter Generation in Kinship" (ICHIGEKI; Noguchi et al, 2009). ICHIGEKI was devised to measure 4-domains of family relationship (Cohesiveness, Power, Interest, and Openness). Noguchi et al. (2009) pointed out that Cohesiveness is most core domain in ICHIGEKI. Cohesiveness was measured for each dyadic relationship by single item which were rated on 6-point Likert-style scale ranging from "very weak" $(=1)$ to "very strong" $(=6)$. In this study, 17 dyadic relationships in three-generation family (see Figure1) were rated with Cohesiveness-Scale.

\section{Procedures}

Data were drawn from 167 Japanese middleclass working mothers. The mean age of the participants was 43.89. Our purpose of this study was to reveal the way to effective family intervention to improve marital relationships. Hence, we selected low marital cohesiveness families based on questionnaire response $(M=4.41)$, and then, 81 families whose score of the marital cohesiveness was in 1-4 ranges were chosen as objects of analyses.

\section{Results}

We conducted four multiple regression analyses on wife-husband cohesiveness. Analysis plan is shown at Figure 2. We gradually added the entering variables to reveal the dyads that significantly relate to marital cohesiveness on four different unit of family system.

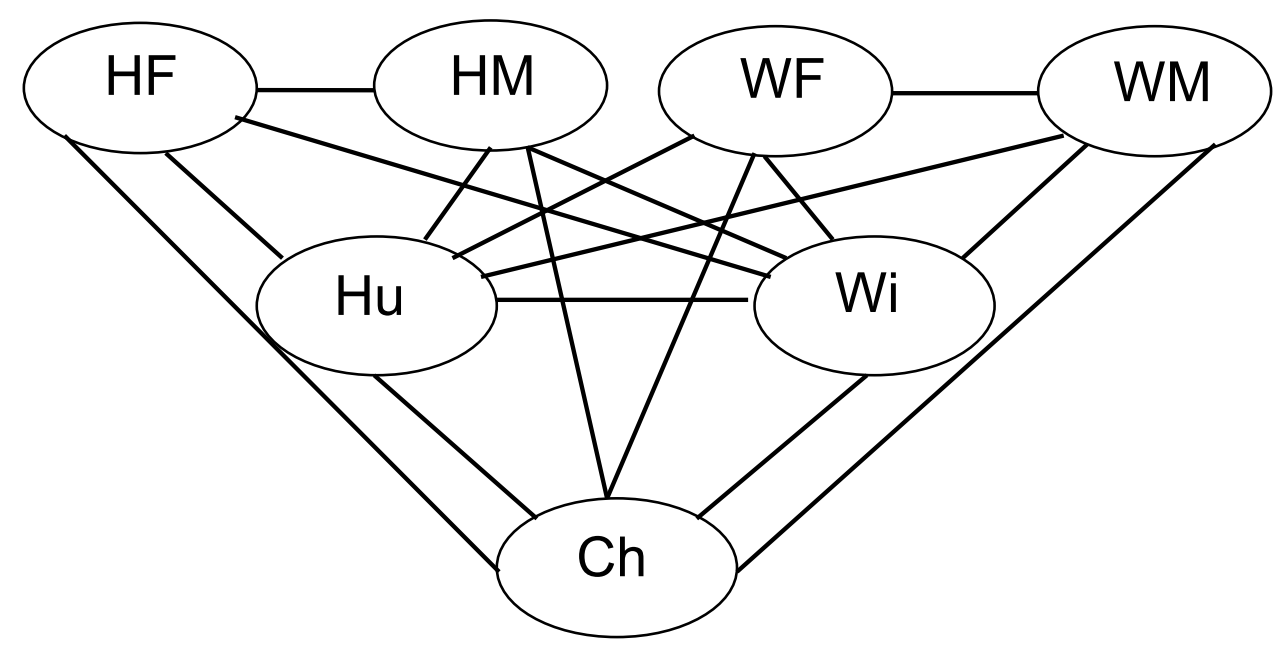

Figure 1.17 dyads in three-generation family

※Note HF: husband's father, HM: husband's mother, Hu: husband,

WF: wife's father, WM: wife's mother, Wi: wife, Ch: child. 


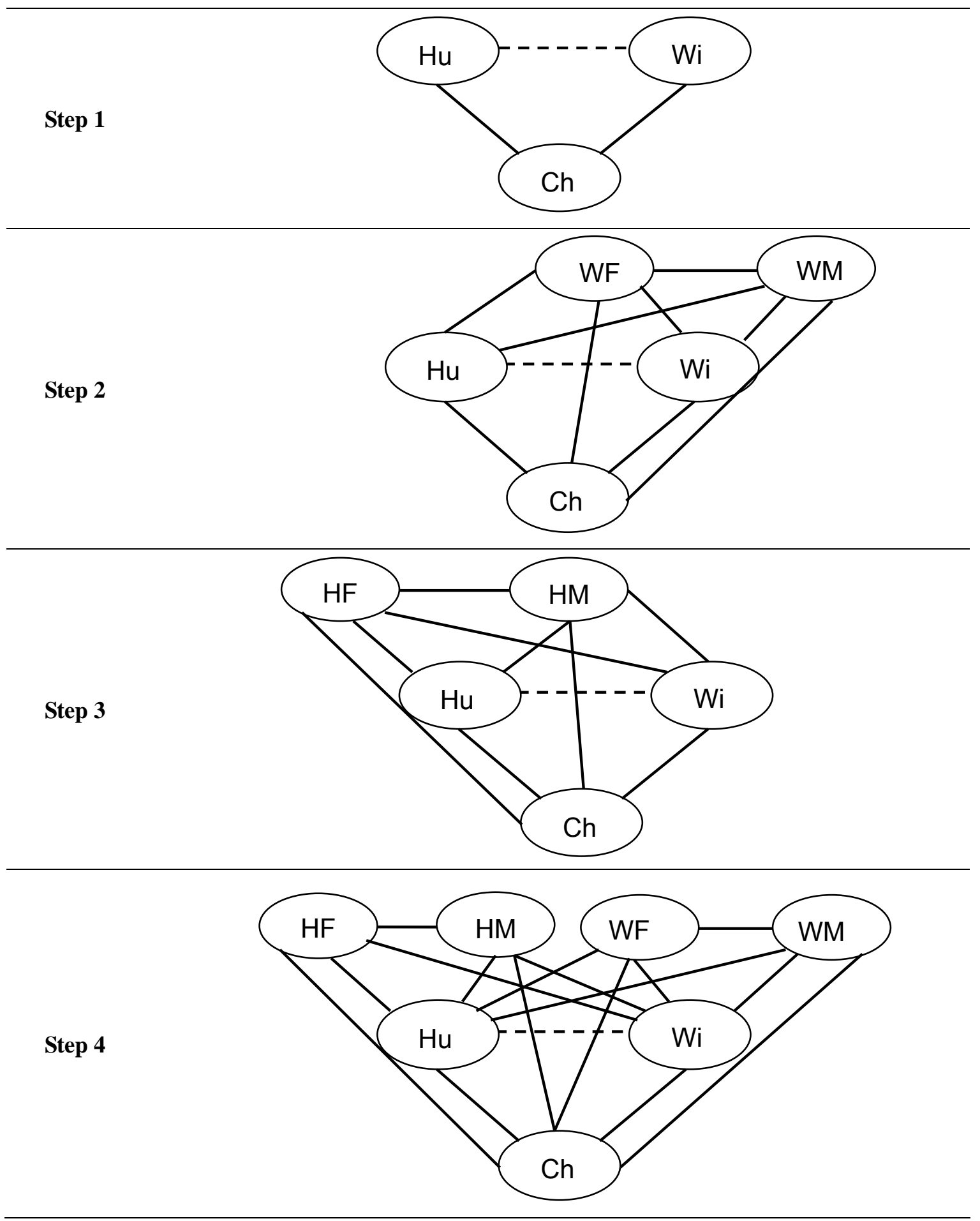

Figure 2. 4 analysis plans of this study

$※$ Note Solid line: Predictor variables, Broken line: Criterion variable

HF: husband's father, HM: husband's mother, Hu: husband,

WF: wife's father, WM: wife's mother, Wi: wife,

Ch: husband's and wife's child. 
First, we conducted multiple regression analysis on marital cohesiveness for analysis1. Predicator variables for analysis1 were shown at Step 1 of Figure 2. We presented the supported regression model at Figure 3.

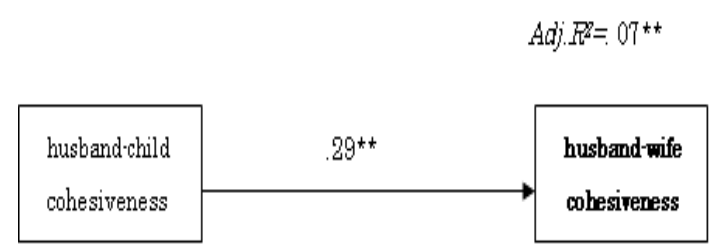

${ }^{\dagger} \not<0.1, * P<05, * * \mathbb{R} 01,{ }^{* * *} \mathbb{R} 001$

Figure 3. The result of analysis 1

Second, we conducted multiple regression analysis on marital cohesiveness for analysis 2 . Predicator variables for analysis 2 were shown at Step 2 of Figure 2. We presented the supported regression model at Figure 4.

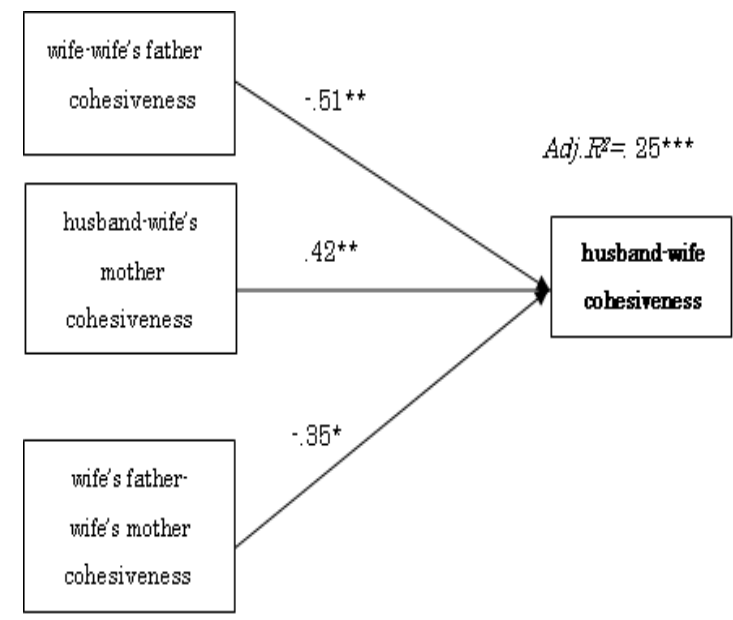

${ }^{1} P<0.1, * P<05,{ }^{* *} \mathbb{R} 01,{ }^{* * *} \mathbb{E} 0.001$

Figure 4 . The result of analysis 2

Third, we conducted multiple regression analysis on marital cohesiveness for analysis 3 .
Predicator variables for analysis3 were shown at Step 3 of Figure 2. We presented the supported regression model at Figure 5.

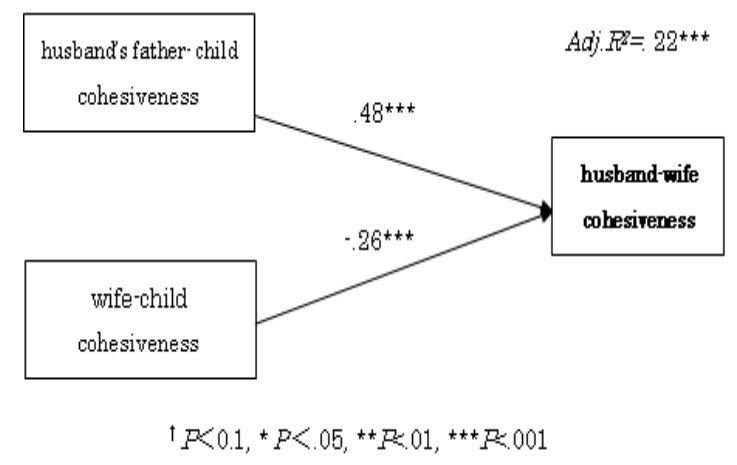

Figure5. The result of analysis 3

Finally, we conducted multiple regression analysis on marital cohesiveness for analysis 3 . Predicator variables for analysis 3 were shown at Step 3 of Figure 2. We presented the supported regression model at Figure 6.

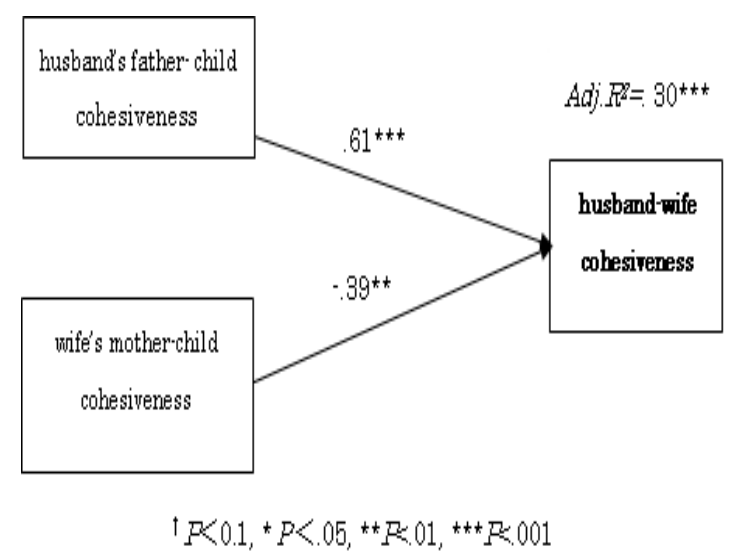

Figure6. The result of analysis 4

\section{Discussions}

In this study, we focused on cohesiveness factor that was suggested as the most core factor in "ICHIGEKI" (Noguchi et al., 2009) and low marital cohesiveness family. 


\section{PREDICATORS FOR MARITAL COHESIVENESS \\ IN THREE-GENERATION FAMILY}

In this study, we revealed dyadic cohesions in three-generation family that relate to marital cohesiveness. Seven dyadic relationships are the significant predicators for marital cohesiveness (see Figure 7 and Figure 8).

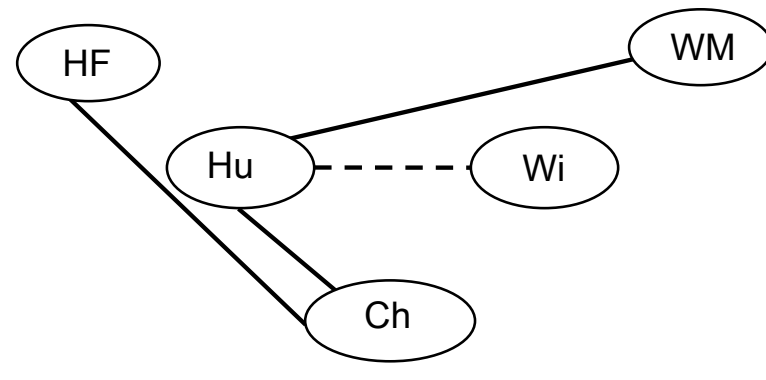

Figure 7. Dyads positively related to marital cohesiveness

$※$ Note Solid line: Predictor variables

Broken line: Criterion variable

HF: husband's father, Hu: husband,

WM: wife's mother, Wi: wife,

Ch: child.

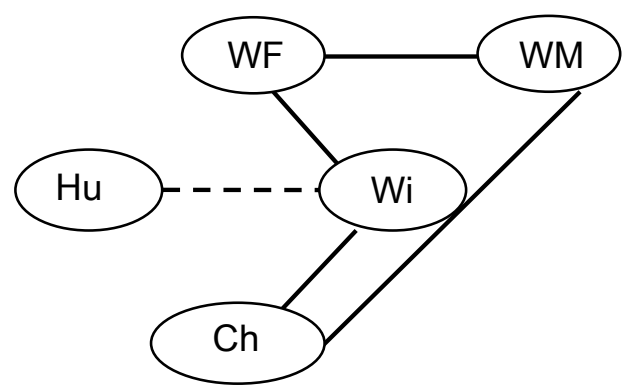

Figure8. Dyads negatively related to marital cohesiveness

※Note Solid line: Predictor variables,

Broken line: Criterion variable

Hu: husband, WF: wife's father,

WM: wife's mother, Wi: wife,

Ch: child.
On account of the space limitation, it is difficult to discuss about all results in this study. Based on our results, we should focus on larger family system (i.e. three generational family) to intervene marital relationship.

Nozue (2008) pointed out that problems between married couples may occur resulted from unsolved problems in their family of origin. However, the results of this study were limited to dyadic cohesiveness between mother and her family of origin. Therefore, Nozue's suggestion can be limitedly applicable to mother's family of origin.

Moreover, the result of this study shows that G1-G3 relationships as well as G1-G2 relationships are important as predictive factors for marital relationships. Given that findings about G2-G3 relationships, it should be cautions to consider two parent-child relationships (husband-child and wife-child) as identical factors for marital relationship.

Future study should explore the nature of the communication, which can determine the quality of dyadic cohesiveness and should examine the causal relationship about those results for proving applicability of clinical intervention.

\section{Acknowledgements}

This study was supported by grants to Koubun Wakashima from Japan society for the Promotion of Science (2010-2011) .

\section{References}

Kawasima, A., Maeshiro, K., Sugawara, M.,

Sakai, A., Ito, K. (2008). Relation between 
adolescence child's cognition to parental conflict and child's depression. Japanese Journal of Educational Psychology, 56(3), 353-363.

Noguchi. S, Kozuka, T., Usami, T., Wakashima, K. (2009). Development and Examination of Validity for a New Family Structure Assessment Scale "ICHIGEKI". Annual Report, Graduate School of Education, Tohoku University, 58(1), 247-265.

Nozue, T. (2008). 夫婦関係の危機之援助. In Nakagama, H., Nozue, T., Nunoshiba, H., Muto, S. (Eds.), Family Psychology. Tokyo: Yuhikaku books, 173-191.

Wakashima, K., Kozuka, T., Itakura, N., \& Usami, T. (2010). Simultaneous and Cumulative Family Relationship: Examining with ICHIGEKI. Interactional Mind III, 92-98. 